\title{
Stripe Systems with Competing Interactions on Quasi-One Dimen- sional Periodic Substrates
}

\author{
Danielle McDermott, ${ }^{a, b}$ Cynthia J. Olson Reichhardt, ${ }^{* a}$ and Charles Reichhardt ${ }^{a}$
}

\author{
Received Xth XXXXXXXXXX 20XX, Accepted Xth XXXXXXXXX 20XX \\ First published on the web Xth $X X X X X X X X X X 200 X$ \\ DOI: 10.1039/b000000x
}

We numerically examine the two-dimensional ordering of a stripe forming system of particles with competing long-range repulsion and short-range attraction in the presence of a quasi-one-dimensional corrugated substrate. As a function of increasing substrate strength or the ratio of the number of particles to the number of substrate minima we show that a remarkable variety of 'distinct orderings can be realized, including modulated stripes, prolate clump phases, two dimensional ordered kink structures, crystalline void phases, and smectic phases. Additionally in some cases the stripes align perpendicular to the substrate troughs. Our results suggest that a new route to self assembly for systems with competing interactions can be achieved through the addition of a simple periodic modulated substrate.

\section{Introduction}

There are a wide variety of systems that exhibit pattern for'mation in the form of ordered stripes, which can often be attributed to the existence of competing interactions $\underline{\underline{1}-\underline{\underline{8}}}$. Such stripe morphologies appear in soft matter systems such as colloids $3,4,8-13$ and lipid monolayers near critical points 14 ; in magnetic systems $\frac{15}{5}$; in certain superconducting vortex systems such as low- $\kappa$ materials $\frac{16}{}$, multi-layered $\frac{17,18}{}$ supercon'ductors, or multi-band superconductors $\frac{19}{}$; in charge-ordered 'states observed in quantum Hall systems ${ }^{20}$ or cuprate super'conductors 21 ; and in ordered states of dense nuclear matter ${ }^{22}$. Numerous studies have been devoted to understanding what types of particle-particle interactions can give rise to such patterns $-\frac{3-6,23-26}{2}$. Having a clear methodology to control the patterns would be very useful for self-assembly and tailoring specified morphologies for applications.

One aspect of these stripe-forming systems that has received little attention is the effect on the pattern formation of adding a periodic substrate. There are many examples of systems in which the addition of a periodic substrate can induce 'different types of ordering. The substrate may occur naturally at the atomic scale due to molecular ordering at a surface, or a substrate can be imposed using an external field or by nanostructuring or etching the surface. A system of repulsively interacting colloids forms a triangular lattice in the absence of a substrate, but when the colloids are placed on an optically created quasi-one-dimensional (q1D) periodic substrate,

${ }^{a}$ Theoretical Division, Los Alamos National Laboratory, Los Alamos, New Mexico 87545, USA. Fax: 1505606 0917; Tel: 1505665 1134; E-mail: cjrx@lanl.gov

${ }^{b}$ Department of Physics, University of Notre Dame, Notre Dame, Indiana 46556, USA. a number of distinct crystalline and smectic orderings appear as a function of substrate strength or commensurability $27-36$. Similarly, magnetic colloids interacting with a fabricated q1D corrugated surface 37,38 also exhibit crystalline disordered and smectic phases as a function of particle density $\frac{37}{}$. In a superconducting vortex system, when a q1D modulated substrate is created by etching the surface of the superconductor, different types of commensurability effects appear that are correlated with ordered and disordered vortex structures $39-41$.

In this work we examine the two-dimensional ordering of particles with long range repulsion and short range attraction interacting with a periodic q1D substrate. The particular model we examine combines Coulomb repulsion with a short-range exponential attraction between particles. In the absence of a substrate, this system is known to exhibit bubble, stripe, void, and uniform phases which have been well characterized as a function of particle density and the ratio of attraction to repulsion $6,10,16,23,42,43$. We specifically focus on parameter regimes in which the system forms stripes in the absence of a substrate ${ }^{6}$. It might be expected that the addition of a q1D periodic substrate to a stripe system would produce only a limited range of phases since the stripes could simply align with the substrate; however, we find that this system exhibits a remarkably rich variety of distinct phases as a function of substrate strength and the ratio of the particle spacing to the substrate minima spacing. These phases include 2D modulated structures, prolate clump crystals, void crystals, and ordered kink arrays. Additionally the stripes can be aligned perpendicular to the substrate troughs. Our results show that the addition of q1D substrates can be a new route to controlling pattern formation in systems with competing interactions. 

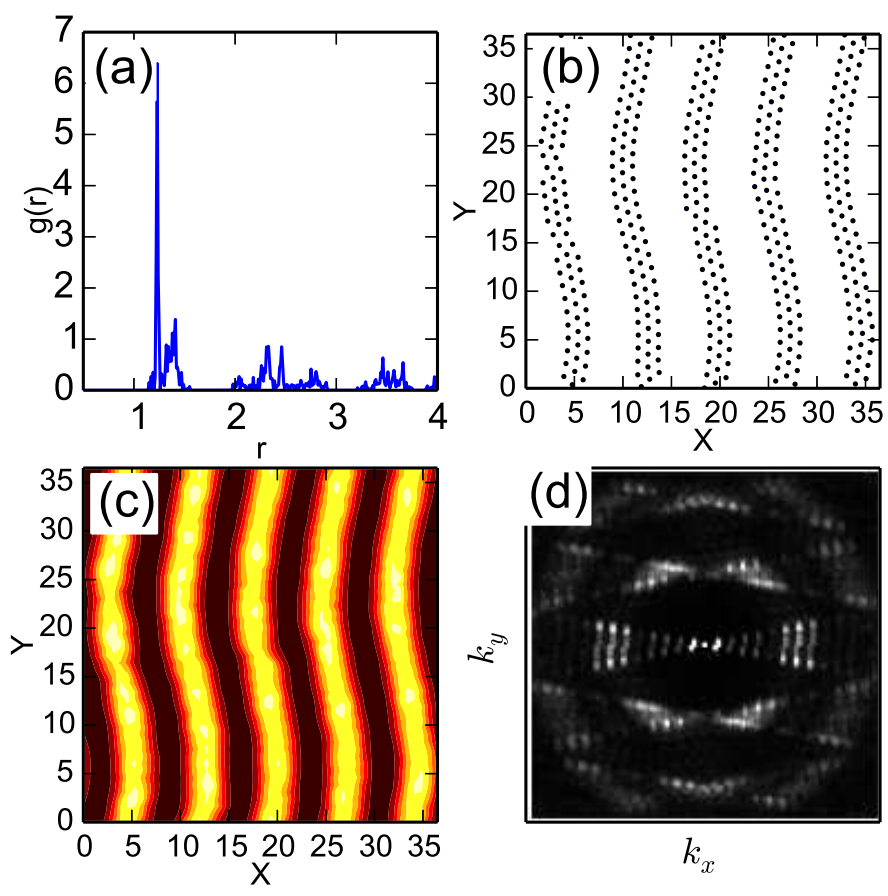

Fig. 1 The stripe system in the absence of a substrate at a density of $\rho=0.3$. (a) Radial distribution function $g(r)$ showing a peak at $r=1.2$. (b) Real space image of the stripes. (c) Density plot in which high densities correspond to brighter spots. (d) $S(k)$.

\section{Simulation}

We consider a two-dimensional system with periodic boundary conditions of size $L \times L$ containing $N$ particles that have pairwise interactions including both repulsive and attractive components. The particle configurations are obtained by annealing the system from a high temperature molten state in small increments to zero temperature. The particle dynamics are governed by the following overdamped equation:

$$
\eta \frac{d \mathbf{R}_{i}}{d t}=-\sum_{j \neq i}^{N} \nabla V\left(R_{i j}\right)+\mathbf{F}_{i}^{s}+\mathbf{F}_{i}^{T} .
$$

Here $\eta$ is the damping term which we set to unity and $\mathbf{R}_{i(j)}$ is the location of particle $i(j)$. The particle-particle interaction potential has the form $V\left(R_{i j}\right)=1 / R_{i j}-B \exp \left(-\kappa R_{i j}\right)$, where $R_{i j}=\left|\mathbf{R}_{i}-\mathbf{R}_{j}\right|$ and $\hat{\mathbf{R}}_{i j}=\left(\mathbf{R}_{i}-\mathbf{R}_{j}\right) / R_{i j}$. The Coulomb term $1 / R_{i j}$ produces a repulsive interaction at long range, while the exponential term gives an attraction at shorter range. At very short range the repulsive Coulomb interaction becomes dominant again. For computational efficiency, we employ a Lekner summation method to treat the long-range Coulomb term ${ }^{44}$. The particle density is $\rho=N / L^{2}$, and unless otherwise noted we take $\rho=0.3$. In the absence of a substrate, previous studies of this model found that for fixed $B=2.0$ and $\kappa=1.0$,

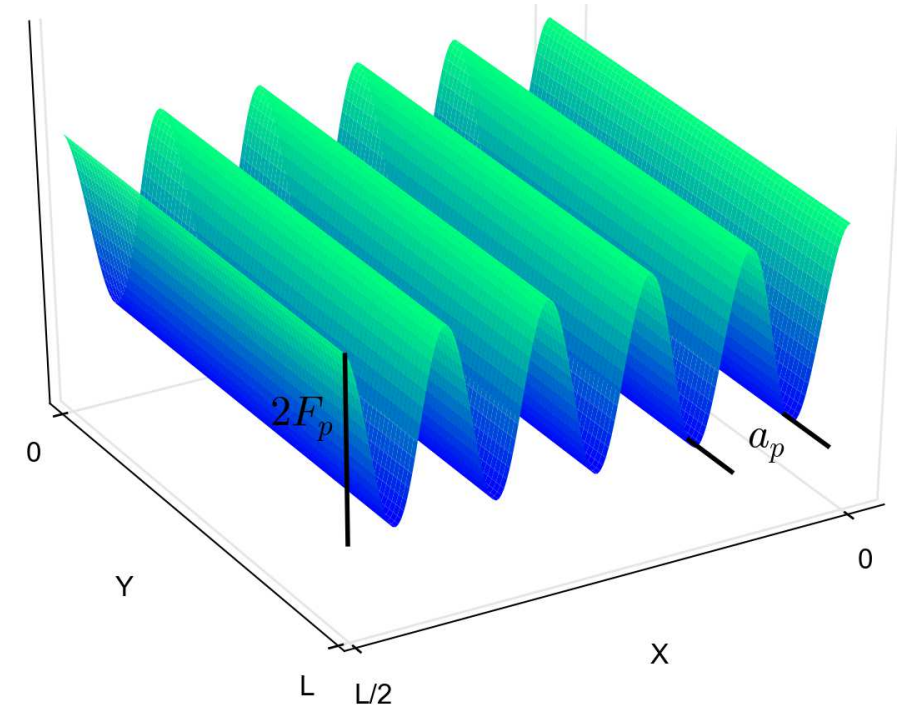

Fig. 2 An example of a q1D periodic pinning substrate used in this work. The potential is modulated in the $x$ direction and the substrate troughs are parallel with the $y$ axis. The total depth of each well is $2 F_{p}$ and the spacing between substrate minima is given by $a_{p}$.

the system initially forms clumps at low density that grow in size up to $\rho=0.27$. For $0.27<\rho \leq 0.46$ the system forms stripes, for $0.46<\rho \leq 0.58$ void crystals form, and a uniform triangular lattice appears for $\rho>0.58^{6}$. Here we fix $B=2.0$ and $\kappa=1.0$ and focus on the stripe regime near the density of $\rho=0.3$ illustrated in Fig.1 (b), where an array of stripes forms with three rows of particles in each stripe. The stripe ordering is also apparent in the corresponding density plot of Fig. 1( c). Figure 11a) shows the radial density function $g(r)$ which has a first neighbor peak at $1.2 a_{0}$, the value of the intra-stripe particle distance $a_{\text {intra. }}$. The structure factor $S(k)$ in Fig. 1(d) has six maxima regions at large $k$ produced by the tendency of the particles to form hexagonal structures within each stripe. The two bright peaks at small $k$ indicate the stripe ordering. For the parameters we consider, the interparticle potential has a minimum at $R_{i j}=1.47 a_{0}$. We focus on systems of size $L=36.5 a_{0}$.

The force from the q1D pinning periodic substrate $\mathbf{F}_{s}$ is given by

$$
\mathbf{F}_{s}=F_{p} \cos \left(2 \pi x / a_{p}\right)
$$

where $a_{p}=L / N_{p}, N_{p}$ is the number of substrate minima, and $a_{p}$ is the spacing between minima. Such a substrate is illustrated in Fig. 2. The pinning force amplitude is $F_{p}$ and we consider values in the range $0.01 \leq F_{p} \leq 6.0$. Our primary interest is in the regime $F_{p}<2.0$ since the transition from particle interaction-dominated to substrate interactiondominated behavior typically occurs within this limit. The thermal force $\mathbf{F}^{T}$ applied during the annealing phase is modeled as Langevin kicks with the properties $\left\langle F^{T}(t)\right\rangle=0$ and 
$\left\langle F_{i}^{T}(t) F_{j}^{T}\left(t^{\prime}\right)\right\rangle=2 \eta k_{B} T \delta_{i j} \delta\left(t-t^{\prime}\right)$. We start from a high temperature liquid state and decrease the temperature in small increments until $T=0$, as in previous studies ${ }^{6}$.

\section{Results}

We first consider the case where the distance $a_{p}$ between the substrate minima is significantly larger than the nearestneighbor particle distance $a_{\text {intra }}$ within a stripe. In Fig. 3 we show the particle positions, $S(k)$, and density plots for a system with $a_{p}=3.65 a_{0}$, giving $a_{p} / a_{\text {intra }}=3.0$. At $F_{p}=0.05$, Fig. 3 a,b,c) indicates that the substrate aligns the stripes along the $y$-direction, parallel to the substrate troughs. For $F_{p}<0.06$ the stripes remain aligned in the $y$ direction and half of the substrate minima contain no particles, since the substrate-free system forms five stripes and there are ten substrate minima. At $F_{p}=0.08$ in Fig. 3 (d,e,f), the stripes have tilted and develop an additional modulated structure in the form of steps. These modulations have a tilted square ordering which can be more clearly seen in the density plot of Fig. 3(f). At $F_{p}=0.2$ in Fig. 3 ( $\mathrm{g}, \mathrm{h}, \mathrm{i})$, the stripes break up and the system forms an array of prolate clumps that have a $2 \mathrm{D}$ periodic ordering. This ordering produces additional features in $S(k)$ at small $k$ values as shown in Fig. 3 $(h)$. The breaking apart of the original stripes permits each of the ten substrate minima to capture an approximately equal number of particles. The clumps exhibit some asymmetry, with the clump width varying from three rows of particles at the center of Fig. 3 $\mathrm{g}$ ) to two rows of particles elsewhere. This produces a smearing of the sixfold ordering at larger values of $k$ in Fig. 3h). At $F_{p}=0.8$ in Fig. 3 j,k,l), stripe ordering returns when the particles form nearly 1D chains stretching along the length of each potential minima. These 1D chains are interspersed with kinks of smaller zig-zag patterns. The kinks have an effective repulsive interaction and tend to form a triangular lattice, as shown in Fig. 31). As $F_{p}$ increases further, the size and number of kinks gradually deceases until the system forms a smectic state of $1 \mathrm{D}$ chains as shown in Fig. 3. 3 ,n,o) at $F_{p}=2.0$. For further increases in $F_{p}$, we find no changes in the smectic structure.

We characterize the onset of the different orderings by measuring the average nearest-neighbor particle distance $\left\langle d_{\min }\right\rangle=$ $N^{-1} \sum_{i=0}^{N} d_{n i}$, where $d_{n i}$ is the distance to the nearest neighbor of particle $i$ as obtained from a Delaunay construction. We also measure $\left\langle d_{p i n}\right\rangle$ which is the average horizontal distance from a particle to the closest substrate minimum, $\left\langle d_{\text {pin }}\right\rangle=$ $N^{-1} \sum_{i=0}^{N}\left(x_{i}-x_{p}\right)$, where $x_{i}$ is the location in the $x$ direction of particle $i$ and $x_{p}$ is the location of the nearest substrate minimum. If all the particles reside at the substrate minima, $\left\langle d_{\text {pin }}\right\rangle=0$. We also measure the total normalized energy of the system $E_{T} / N$. In Fig. 4 we plot $\left\langle d_{\min }\right\rangle,\left\langle d_{\text {pin }}\right\rangle$, and $E_{T} / N$ vs $F_{p}$ for the system in Fig. 3 There is a feature near $F_{p}=0.08$ at the point where the straight stripes
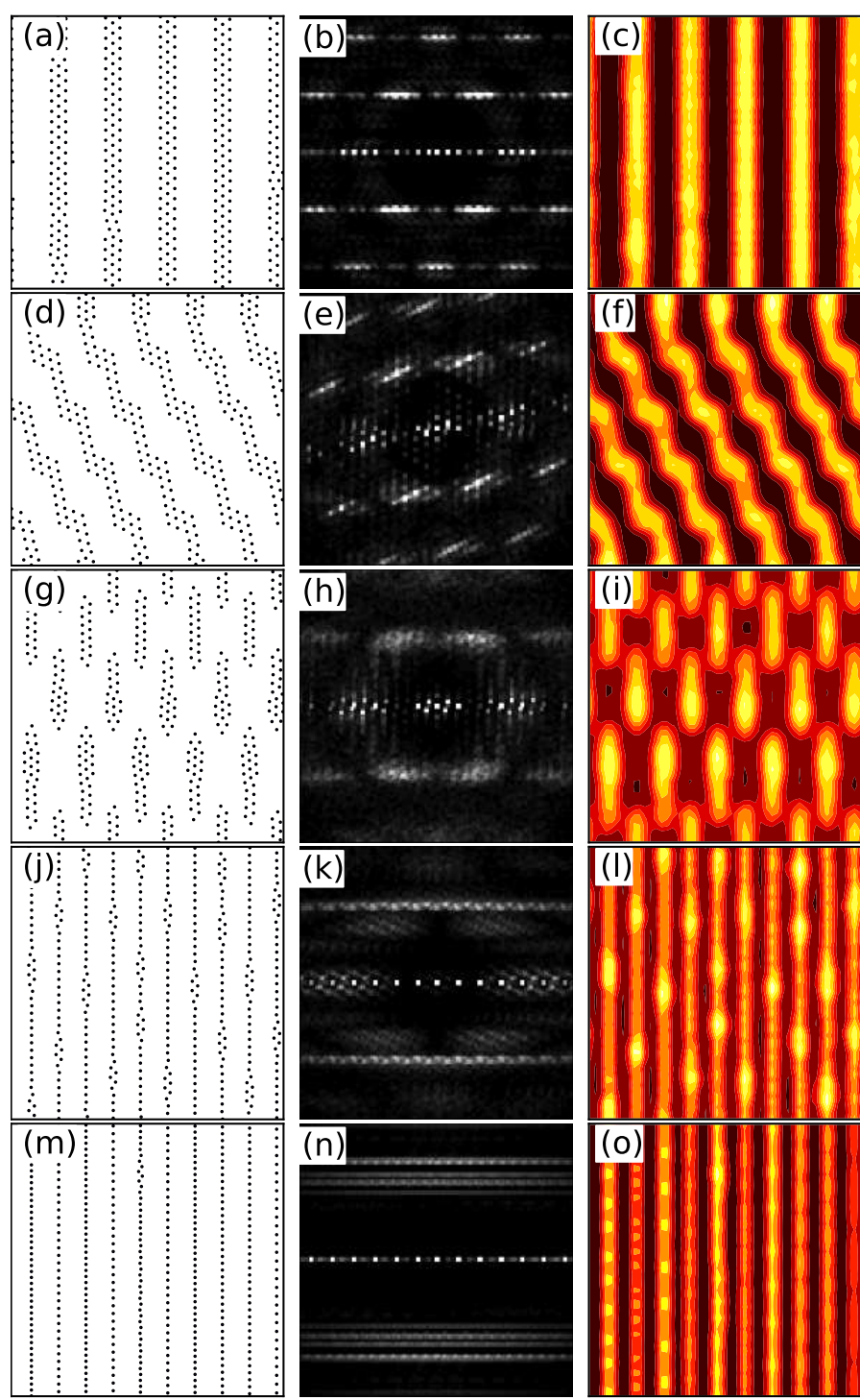

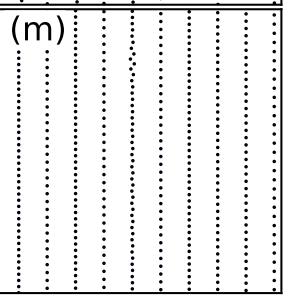

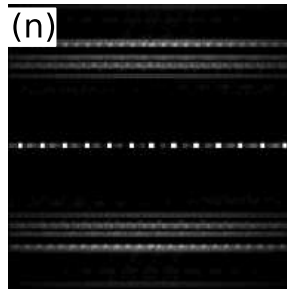

Fig. 3 Real space particle positions (left column), S(k) (central column), and density plots (right column) for stripes ordering on a periodic q1D substrate with troughs aligned in the $y$ direction for a system with $a_{p}=3.65$. (a,b,c) Aligned stripe phase at $F_{p}=0.05$. (d,e,f) Modulated stripes at $F_{p}=0.08$. (g,h,i) Prolate clump phase at $F_{p}=0.2$. (j,k,1) $1 \mathrm{D}$ kink phase at $F_{p}=0.8$. (m,n,o) Smectic phase at $F_{p}=2.0$. 


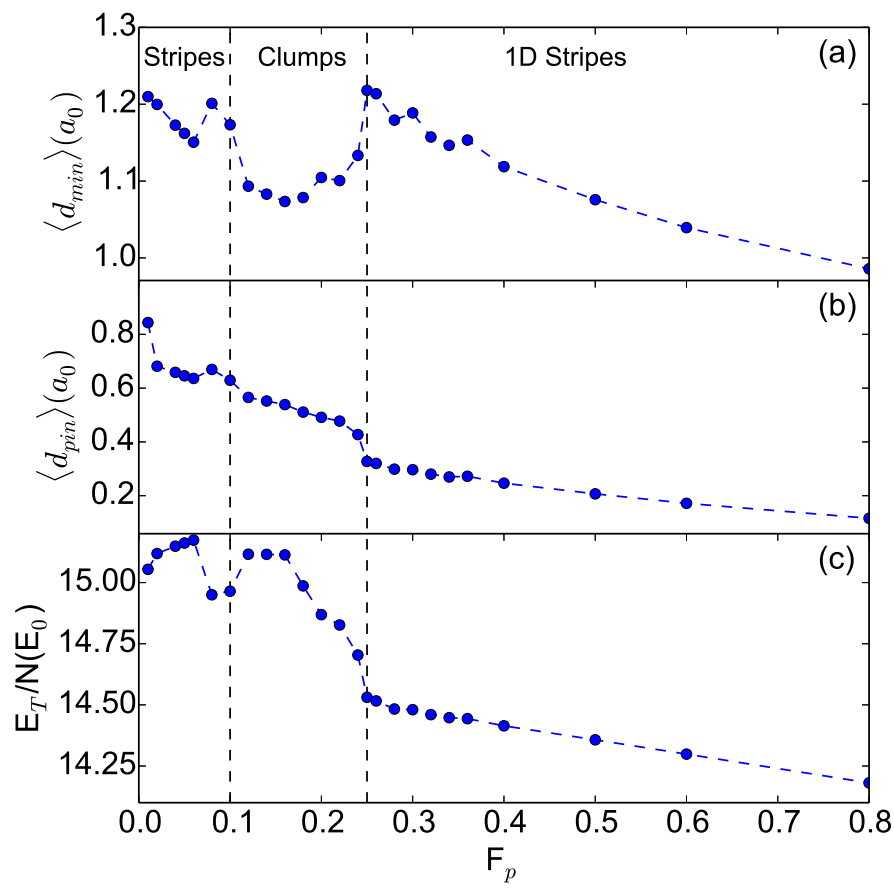

Fig. 4 (a) Nearest neighbor distance $\left\langle d_{\min }\right\rangle$, (b) horizontal distance to closet substrate minima $\left\langle d_{\text {pin }}\right\rangle$, and (c) total energy of the system $E_{T} / N$ vs $F_{p}$ for the system in Fig. 3. highlighting the changes in the patterns.

shown in Fig. 3 a,b,c) transition to the modulated stripe phase shown in Fig. 3 d,e,f). As $F_{p}$ further increases, the modulated stripes gradually transform into the clump phase shown in Fig. 3(g,h,i). Near $F_{p}=0.28$ we find a signature of the transition from the clumps to the 1D kinked stripe state shown in Fig. $3(\mathrm{j}, \mathrm{k}, \mathrm{l})$ in the form of a peak in $\left\langle d_{\min }\right\rangle$, a dip in $\left\langle d_{\text {pin }}\right\rangle$, and a cusp in $E_{T} / N$. For $F_{p}>0.28$ the curves are smooth as the number of kinks gradually decreases and the particles move closer to the substrate minima. This is indicated by the steady decrease of $\left\langle d_{p i n}\right\rangle$ which approaches zero as the system forms the fully smectic state shown in Fig. 3 3 m,n,o).

For the same set of parameters but larger $a_{p}$ we observe the same set of patterns. If we increase the particle density $\rho$ but hold the substrate period fixed, new patterns appear. At higher $\rho$ the prolate clump phase is lost but new types of modulated kink phases occur. Fig. 5 shows the real space, $S(k)$, and density plots for a system with $a_{p}=3.65 a_{0}$ at a particle density of $\rho=0.363$ where the substrate-free system still forms stripes. The increase in particle density makes it more difficult to compress the particles into the 1D patterns observed at $\rho=0.3$ in Fig. 3 Figure $5(\mathrm{a}, \mathrm{b}, \mathrm{c})$ shows the ordering for $F_{p}=0.2$, where a modified stripe phase containing a semiperiodic array of spokes appears. At $F_{p}=0.3$, Fig. 5 (d,e,f) shows that an ordered array of kinks forms where each substrate minimum
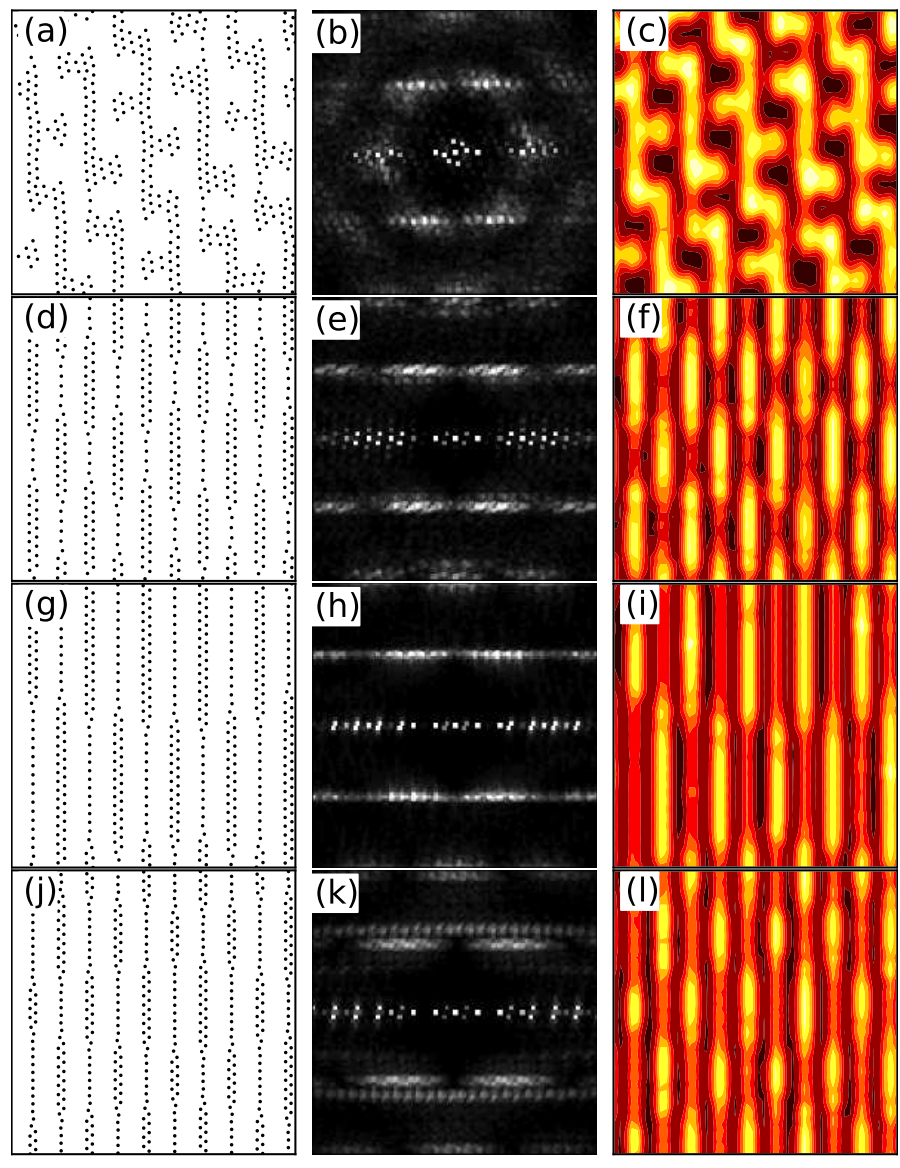

Fig. 5 Real space particle positions (left column), $S(k)$ (central column), and density plots (right column) for a periodic q1D substrate with $a_{p}=3.65 a_{0}$ at $\rho=0.363$. (a,b,c) Modulated stripe phase at $F_{p}=0.2$. (d,e,f) Ordered kink phase at $F_{p}=0.3$. (g,h,i) Ordered kink phase at $F_{p}=0.5$. (j,k,l) Ordered kink phase at $F_{p}=1.0$. 

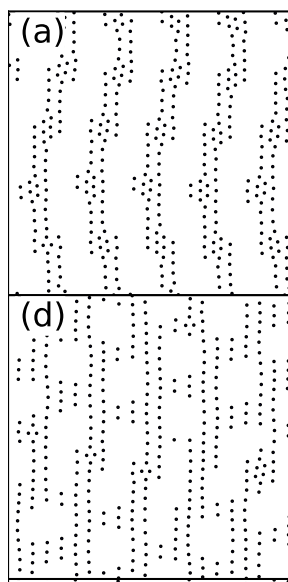

(g)
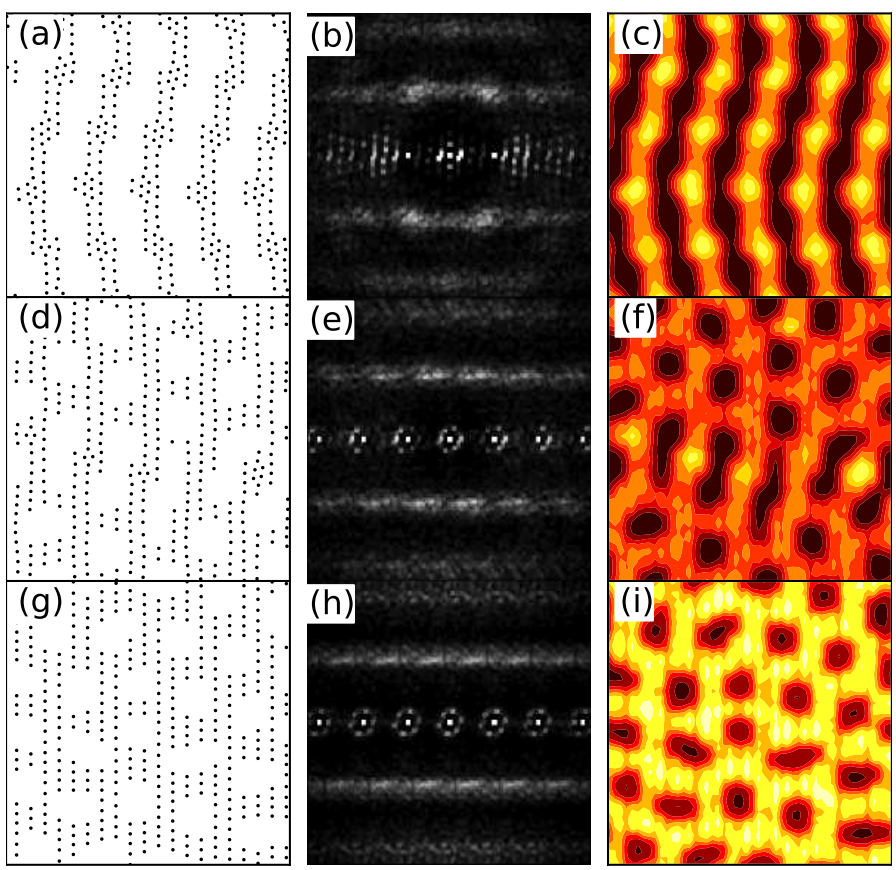

Fig. 6 Real space particle positions (left column), $S(k)$ (central column), and density plots (right column) for a periodic q1D substrate with $a_{p}=1.82$. (a,b,c) Modulated stripe phase with a 2D periodic array of bubbles at $F_{p}=0.14$. (d,e,f) Void phase at $F_{p}=0.2$. $(\mathrm{g}, \mathrm{h}, \mathrm{f})$ A better-defined void phase at $F_{p}=2.0$.

contains regions of two rows of particles interspersed with regions that are only a single row wide. The density plot in Fig. 5(f) indicates that the kinks order into a periodic structure. As $F_{p}$ increases the number of kinks changes, as shown in Fig. 5 $5 \mathrm{~g}, \mathrm{~h}, \mathrm{i})$ for $F_{p}=0.5$. As $F_{p}$ is further increased the system gradually develops more $1 \mathrm{D}$ behavior as illustrated in Fig. $\left[5(\mathrm{j}, \mathrm{k}, \mathrm{l})\right.$ at $F_{p}=1.0$. For high enough $F_{p}$, all of the kinks vanish.

We next consider the limit in which the spacing $a_{p}$ between substrate minima becomes comparable to or smaller than the average nearest-neighbor particle spacing $a_{\text {intra }}$. In Fig. 6 we plot the real space particle positions, $S(k)$, and the local density for samples with $a_{p}=1.82$ and $a_{p} / a_{\text {intra }}=1.5$ for varied $F_{p}$. We find that when the pinning density is high, the original stripe structure remains intact up to relatively large values of $F_{p}$ since the smaller substrate spacing permits all of the particles to take advantage of substrate minimum locations while still remaining in the original stripe pattern. Above this point, as $F_{p}$ is increased we observe a modulated stripe phase with square ordering as shown in Fig. 6 a,b,c) for $F_{p}=0.14$. At higher $F_{p}$ there is a transition to a void crystal of the type shown in Fig. 6(d,e,f) for $F_{p}=0.2$. This void crystal becomes more stable and persists as $F_{p}$ is further increased, as illus-

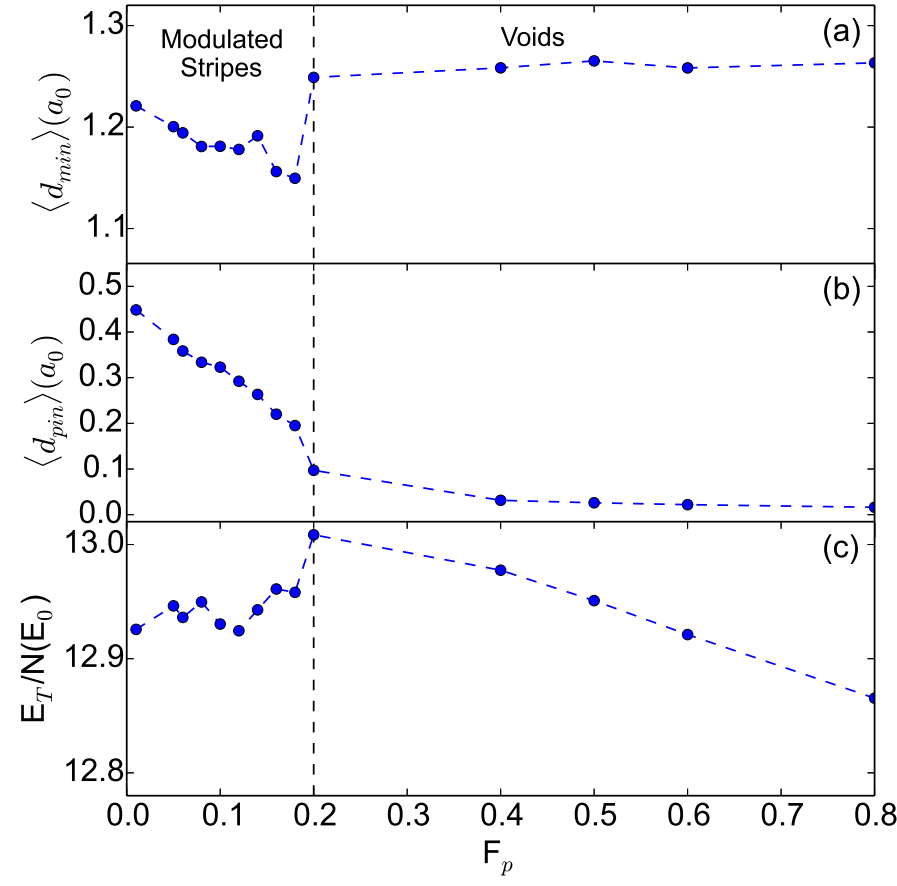

Fig. 7 (a) $\left\langle d_{\text {min }}\right\rangle$, (b) $\left\langle d_{\text {pin }}\right\rangle$, and $E_{T} / N$ vs $F_{p}$ for the system in Fig. 6 showing the onset of the different phases. S: stripe phase; MS: modulated stripe phase.

trated in Fig. 6( g,h,i) for $F_{p}=2.0$. In Fig. 7( $(\mathrm{a}, \mathrm{b}, \mathrm{c})$ we plot the corresponding values of $\left\langle d_{\min }\right\rangle,\left\langle d_{p i n}\right\rangle$, and $E_{T} / N$ versus $F_{p}$ for the system in Fig. 6. At $F_{p}=0.1$, there is an inflection in $\left\langle d_{\min }\right\rangle$ at the transition from the stripe to the modulated stripe phase. The onset of the void phase near $F_{p}=0.2$ is marked by features in $\left\langle d_{\min }\right\rangle$. Once the voids have formed, they remain stable for increasing $F_{p}$ since all the particles can fit in a potential minimum. We observe similar void formation when we fix $a_{p}=1.82$ but vary the particle density $\rho$.

When the pinning density is increased, the stripe state persists to higher values of $F_{p}$. The stripe alignment, however, alters and we find that the stripes generally run perpendicular to the direction of the substrate troughs when $a_{p}$ is small, as shown in in Fig. $8(\mathrm{a}, \mathrm{b}, \mathrm{c})$ for $F_{p}=2.0, a_{p}=1.2$, and $a_{p} / a_{\text {inter }}=1.0$. When $a_{p}<a_{\text {inter }}$ we observe a transition from the stripe phase to a clump phase as illustrated in Fig. $8($ d,e,f) at $F_{p}=0.8$ for a system with $a_{p} / a_{\text {inter }}=0.76$. In general, for $a_{p} / a_{\text {inter }}<1.0$, clump phases form at large $F_{p}$.

\section{SUMMARY}

We examine a stripe forming system interacting with a periodic quasi-one dimensional substrate. We show that as a function of substrate strength and density, a remarkably rich variety of distinct orderings can be realized. These phases include 

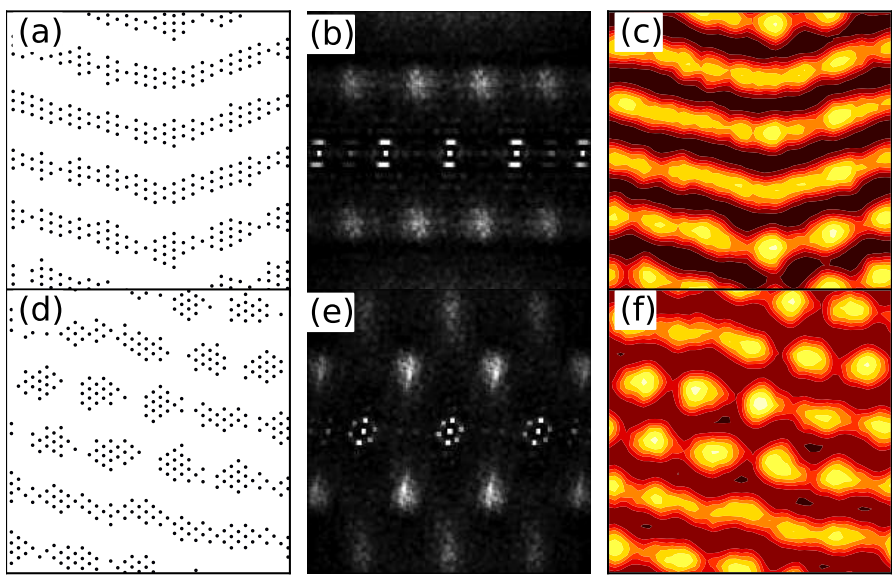

Fig. 8 Real space particle positions (left column), $S(k)$ (central column), and density plots (right column) for periodic q1D substrates. (a,b,c) A perpendicular stripe at $a_{p}=1.2$ and $F_{p}=2.0$. (d,e,f) A clump phase at $a_{p}=0.9125$ and $F_{p}=0.8$.

stripes containing modulations that themselves form a 2D ordered structure, prolate clump phases, various types of 2D ordered kink arrays, and smectic structures. For denser substrate arrays we observe transitions from a modulated stripe phase to a void crystal or a clump phase. Our results show that corrugated substrates could provide a possible new route to controlling pattern forming systems.

\section{Acknowledgements}

This work was carried out under the auspices of the NNSA of the U.S. DoE at LANL under Contract No. DE-AC5206NA25396.

\section{References}

1 M. Seul and D. Andelman, Science, 1995, 267, 476.

2 A.D. Stoycheva and S.J. Singer, Phys. Rev. Lett., 2000, 84, 4657.

3 G. Malescio and G. Pellicane, Nature Mater., 2003, 2; Phys. Rev. E, 2004, 70, 021202.

4 M.A. Glaser, G.M. Grason, R.D. Kamien, A. Kosmrlj, C.D. Santangelo, and P. Ziherl, EPL, 2007, 78, 46004.

5 E. Edlund and M.N. Jacobi, Phys. Rev. Lett., 2010, 105, 137203.

6 C.J. Olson Reichhardt, C. Reichhardt, and A.R. Bishop, Phys. Rev. E, 2010, 82, 041502.

7 A. Giuliani, J.L. Lebowitz, and E.H. Lieb, Phys. Rev. B, 2011, 84, 064205.

8 N.G. Almarza, J. Pekalski, and A. Ciach, J. Chem. Phys., 2014, 140, 164708 .

9 A. Imperio, L. Reatto, and S. Zapperi, Phys. Rev. E, 2008, 78, 021402.

10 Y.H. Liu, L.Y. Chew, and M.Y. Yu, Phys. Rev. E, 2008, 78, 066405.

11 J.-X. Chen, J.-W. Mao, S. Thakur, J.-R. Xu, and F.-Y. Liu, J. Chem. Phys., 2011, 135, 094504.

12 H.J. Zhao, V.R. Misko, and F.M. Peeters, New J. Phys., 2012, 14, 063032.
13 L.Q. Costa Campos, S.W.S. Apolinario, and H. Löwen, Phys. Rev. E, 2013, 88, 042313.

14 S.L. Keller and H.M. McConnell, Phys. Rev. Lett., 1999, 82, 1602.

15 M. Seul and R. Wolfe, Phys. Rev. A, 1992, 46, 7519.

16 X.B. Xu, H. Fangohr, S.Y. Ding, F. Zhou, X.N. Xu, Z.H. Wang, M. Gu, D.Q. Shi, and S.X. Dou, Phys. Rev. B, 2011, 83, 014501.

17 E. Babaev and M. Speight, Phys. Rev. B, 2005, 72, 180502(R).

18 C.N. Varney, K.A.H. Sellin, Q.-Z. Wang, H. Fangohr, and E. Babaev, J. Phys.: Condens. Matter, 2013, 25, 415702; L. Komendova, M.V. Milosevic, and F.M. Peeters, Phys. Rev. B, 2013, 88, 094515.

19 J. Gutierrez, B. Raes, A.V. Silhanek, L.J. Li, N.D. Zhigadlo, J. Karpinski, J. Tempere, and V.V. Moshchalkov, Phys. Rev. B, 2012, 85, 094511; J.A. Drocco, C.J. Olson Reichhardt, C. Reichhardt, and A.R. Bishop, J. Phys.: Condens. Matter, 2013, 25, 345703.

20 M.M. Fogler, A.A. Koulakov, and B.I. Shklovskii, Phys. Rev. B, 1996, 54, 1853; R. Moessner and J.T. Chalker, ibid., 1996, 54, 5006; E. Fradkin and S.A. Kivelson, ibid., 1999, 59, 8065; J. Gores, G. Gamez, J.H. Smet, L. Pfeiffer, K. West, A. Yacoby, V. Umansky, and K. von Klitzing, Phys. Rev. Lett., 2007, 99, 246402.

21 J. Zaanen and O. Gunnarsson, Phys. Rev. B, 1989, 40, 7391; V. Emery S. Kivelson, and J. Tranquada, Proc. Natl. Acad. Sci. U.S.A., 1999, 96, 8814.

22 G. Watanabe, T. Maruyama, K. Sato, K. Yasuoka, and T. Ebisuzaki, Phys. Rev. Lett, 2005, 94, 031101.

23 C. Reichhardt, C.J. Olson, I. Martin, and A.R. Bishop, Europhys. Lett., 2003, 61, 221. C.J. Olson Reichhardt, C. Reichhardt, I. Martin, and A.R. Bishop, Physica D, 2004, 193, 303.

24 E. Edlund, O. Lindgren, and M.N. Jacobi, Phys. Rev. Lett., 2011, 107, 085501.

25 K.A.H. Sellin and E. Babaev, Phys. Rev. E, 2013, 88, 042305.

26 G. Zhang, F.H. Stillinger, and S. Torquato, Phys. Rev. E, 2013, 88, 042309.

27 A. Chowdhury, B.J. Ackerson, and N.A. Clark, Phys. Rev. Lett., 1985, 55, 833.

28 J. Chakrabarti, H.R. Krishnamurthy, A.K. Sood, and S. Sengupta, Phys. Rev. Lett., 1995, 75, 2232.

29 Q.-H. Wei, C. Bechinger, D. Rudhardt, and P. Leiderer, Phys. Rev. Lett., 1998, 81, 2606.

30 L. Radzihovsky, E. Frey, and D.R. Nelson, Phys. Rev. E, 2001, 63, 031503.

31 C. Reichhardt and C.J. Olson Reichhardt, Phys. Rev. E, 2005, 72, 032401.

32 P. Chaudhuri, C. Das, C. Dasgupta, H.R. Krishnamurthy, and A.K. Sood, Phys. Rev. E, 2005, 72, 061404; M. Schmiedeberg, J. Roth, and H. Stark, Phys. Rev. Lett., 2006, 97, 158304.

33 W. Strepp, S. Sengupta, and P. Nielaba, Phys. Rev. E, 2002, 66, 056109; F. Burzle and P. Nielaba, Phys. Rev. E, 2007, 76, 051112.

34 J. Baumgartl, M. Brunner, and C. Bechinger, Phys. Rev. Lett., 2004, 93, 168301.

35 C. Dalle-Ferrier, M. Kruger, R.D.L. Hanes, S. Walta, M.C. Jenkins, and S.U. Egelhaaf, Soft Matter, 2011, 7, 2064.

36 L. Zaidouny, T. Bohlein, R. Roth and C. Bechinger, Soft Matter, 2013, 9, 9230.

37 J. Hu and R.M. Westervelt, Phys. Rev. B, 1996, 55, 771.

38 P. Tierno, Phys. Rev. Lett., 2012, 109, 198304.

39 O. Daldini, P. Martinoli, J.L. Olsen and G. Berner, Phys. Rev. Lett., 1974, 32, 218; P. Martinoli, H. Beck, M. Nsabimana and G.-A. Racine, Physica $B, 1981, \mathbf{1 0 7}, 455$.

40 L.S. Levitov, Phys. Rev. Lett., 1991, 66, 224

41 Q. Lu, C.J. Olson Reichhardt, and C. Reichhardt, Phys. Rev. B, 2007, 75, 054502.

42 F.F. Munarin, K. Nelissen, W.P. Ferreira, G.A. Farias, and F.M. Peeters, Phys. Rev. E, 2008, 77, 031608.

43 C. Reichhardt, C.J. Olson Reichhardt, I. Martin, and A.R. Bishop, Phys.

6 | Journal Name, 2010, [vol],1-7 
Rev. Lett., 2003, 90, 026401; C.J. Olson Reichhardt, C. Reichhardt, and A.R. Bishop, Phys. Rev. E, 2011, 83, 041501.

44 J. Lekner, Physica A, 1991, 176, 485; N. Grønbech-Jensen, G. Hummer, and K.M. Beardmore, Mol. Phys., 1997, 92, 941; M. Mazars, J. Chem. Phys., 2001, 115, 2955. 